\title{
REAL TIME PCR ASSAY FOR THE DIFFERENTIATION OF MYCOBACTERIAL SPECIES IN BRONCHIAL WASHINGS
}

\author{
${ }^{1}$ T.P. Keerthirathne, ${ }^{1 *}$ D.N. Magana-Arachchi, ${ }^{2}$ S.D.S.S. Sooriyapathirana and \\ ${ }^{3}$ R.M.D. Madegedara \\ ${ }^{l}$ National Institute of Fundamental Studies, Hantana road, Kandy, Sri Lanaka \\ ${ }^{2}$ Department of Molecular Biology and Biotechnology, University of Peradeniya, Sri Lanka \\ ${ }^{3}$ Respiratory Disease Treatment Unit \& Teaching Hospital, Kandy, Sri Lanka
}

\begin{abstract}
Pulmonary infections caused by Nontuberculous Mycobacteria (NTM) species has to be carefully interpreted due to their ubiquitous nature. NTM infections are more common than before in nonimmunosuppressed hosts. Real-time PCR designed for Mycobacterium species, allows precise identification through melting point analysis. This study was designed for identification of Mycobacterium species present in bronchial washings. Ethical clearance was obtained from the PostGraduate Institute of Science, University of Peradeniya. Bronchial washings ( $n=150)$ were collected from patients, suspected of having pulmonary diseases, attending the General Hospital Kandy. The samples were processed according to modified Petroff's method and inoculated onto LöwensteinJensen medium. Culture positives were subjected to Ziehl-Neelsen (ZN) staining, DNA were extracted from AFB isolates using the standard CTAB (N Cetyl-N, N, N-trimethyl ammonium bromide) method. SYBR green mediated real-time PCR assay was conducted to identify rapid and slow growers in two parallel reactions. Primers specific for Mycobacterium genus, Mycobacterium tuberculosis complex (MTC), M. avium complex (MAC), M. chelonae- M. abscessus group (MCAG) and M. fortuitum group (MFG) were used. Among the 26 AFB isolates 25 were found to be belonging to the Mycobacterium genus. Two MTC isolates and three MAC isolates were confirmed; following reaction I. Reaction II confirmed the presence of Mycobacterium genus and the presence of MCAG for two isolates. Application of SYBR green mediated real time PCR assay in clinical microbiology could improve the diagnostics due to the increased specificity. Moreover, it is a tool that can be used for the rapid detection of pathogenic NTM species.
\end{abstract}

Keywords: NTM, SYBR green, real-time PCR, AFB

\section{INTRODUCTION}

The genus Mycobacterium includes non-tuberculous mycobacteria (NTM) apart from Mycobacterium tuberculosis (MTB) [1]. NTM are diverse, ubiquitous organisms that can found in the environment, including in water and soil. NTM organisms are well known for its infrequent infection on both immunocompetent and immunocompromised individuals [2]. Accurate detection and identification of NTM is important to the species level because patients with NTM infections show clinical signs that are similar to those of patients with tuberculosis (TB), causing clinical misleading during therapeutic actions [3]. Nevertheless, the presence of NTM in the patients with TB can have a considerable influence on clinical management because incorrect diagnosis of pulmonary TB due to the presence of NTM can lead to unsuitable and unnecessary treatment of patients with NTM infections [4] which could lead to the emergence of drug resistant Mycobacterium strains. Thus, it's important to rapidly distinguish NTM from Mycobacterium tuberculosis complex (MTC) to administer appropriate treatment.

Different methods are used for the identification of NTM in different regions of the world. Even though, most laboratories use conventional methods, improved molecular methods have been reported as having the ability to differentiate and identify more

Corresponding Author Email: *cellbiology@ifs.ac.lk 
NTM species rapidly [5]. Espy M. J. et al (2006) states that "Real-time PCR provide equivalent sensitivity and specificity as conventional PCR combined with Southern blot analysis" [6]. Moreover, when compared with conventional PCR methods, as the nucleic acid amplification and detection steps are performed in the same closed system the possibility for contamination by the release of the amplicon is neglectful. Speedy detection is also an added advantage in the use of real-time PCR technique. The objective of the study was to identify the Mycobacterium species present in bronchial washings using SYBR Green mediated real time PCR.

\section{METHODOLOGY}

Ethical clearance was obtained from the Postgraduate Institute of Science, University of Peradeniya, Sri Lanka. Bronchial washings $(n=150)$ from the patients suspected of having pulmonary infections, were obtained from the General Hospital Kandy, Sri Lanka. The samples were liquefied and decontaminated with $4 \% \mathrm{NaOH}$ according to the modified Petroff's method and was inoculated on to Löwenstein-Jensen medium (L-J medium), LJ media containing Thiophene -2 carboxylic acid hydrazide $(\mathrm{TCH})$ and p-nitrobenzioc acid (PNB) and incubated at $37{ }^{\circ} \mathrm{C}$ and at $28{ }^{\circ} \mathrm{C}$ respectively. Inoculated PNB containing cultured media were incubated under light and dark conditions. The inoculated culture tubes were incubated for 8 to 12 weeks. When the growth was detected, Ziehl-Neelsen stain (ZN stain) was carried out in order to confirm the presence of acid fast organisms. DNA was extracted from confirmed acid fast bacilli (AFB) positive isolates according to the standard CTAB (N-Cetyl-N, N, N-trimethyl ammonium bromide) method [7].

Primers targeting the internal transcribed spacers (ITs) of MTC and MCAG (M. chelonae-M. abscessus group), the 16S rRNA genes of MAC (M. avium complex), MFG (M. fortuitum group) and Mycobacterium genus were used for the real time multiplex, PCR assay. Amplification was monitored by the measurement of the SYBR Green fluorescence. Subsequent to the cycling process melting curves were generated by inclining the temperature from $60{ }^{\circ} \mathrm{C}$ to $95{ }^{\circ} \mathrm{C}$ at $0.2{ }^{\circ} \mathrm{C} / \mathrm{s}[8]$.

The real time multiplex, PCR assay was conducted in two separate reactions where primers specific for MTC and MAC were in reaction I and primers specific for MCAG and MFG were in reaction II which helped in identifying slow and rapid growers respectively (Table 01). Primers targeting AFB genus were included in both the reactions. Each reaction was carried out in a $25 \mu \mathrm{l}$ volume which contained $2.0 \mu \mathrm{l}$ of $25 \mathrm{mM} \mathrm{MgCl} 2,0.25 \mu \mathrm{l}$ of $5 \mathrm{u} / \mu 1 \mathrm{Taq}$ polymerase, $5.0 \mu 1$ of $5 X$ PCR Buffer, $2.5 \mu 1$ of $1 \mathrm{mM}$ dNTP mix and $1.0 \mu 1$ of each primer $(10 \mu \mathrm{m})$. The PCR amplification process was initiated by ramping the temperature at $95{ }^{\circ} \mathrm{C}$ for 5 minutes followed by 40 cycles of the amplification process $\left(95^{\circ} \mathrm{C}\right.$ for $15 \mathrm{~s}, 60$ ${ }^{0} \mathrm{C}$ for 30 s and $72{ }^{\circ} \mathrm{C}$ for 30 s).

Table 1: Primer sequences which were used for the study

\begin{tabular}{|c|c|c|c|}
\hline Organism & $\begin{array}{l}\text { Target } \\
\text { region }\end{array}$ & Primer sequence & Reaction \\
\hline MAC & $16 \mathrm{~S}$ & $\begin{array}{l}\text { F:CCTCAAGACGCATGTCTTC } \\
\text { F:GACCTTTAGRCGCATGTCTTT } \\
\text { R: ACCTACCGTCAATCCGAGAA }\end{array}$ & I \\
\hline MTC & ITS & $\begin{array}{l}\text { F: GCGAGAGCCGGGTGCATG } \\
\text { R: AACAGTGTGTTGGTGGCCAA }\end{array}$ & I \\
\hline Mycobacterium genus & $16 \mathrm{~S}$ & $\begin{array}{l}\text { F: CCGCAAGRCTAAAACTCAAA } \\
\text { R: TGCACACAGGCCACAAGGGA }\end{array}$ & I / II \\
\hline MCAG & $16 \mathrm{~S}$ & $\begin{array}{l}\text { F: TAAGGAGCACCATTTCCCAG } \\
\text { R: CGACGTTTTGCCGACTAACC }\end{array}$ & II \\
\hline
\end{tabular}


MFG

\section{RESULTS}

Following the sample processing and culture, 55 samples yielded positive cultures. However, only 26 were AFB positive. The SYBR green mediated real time multiplex PCR assay confirmed the presence of
Mycobacterium genus in $25 \mathrm{AFB}$ positive isolates. Reaction I of the real time multiplex PCR assay confirmed the presence of AFB genus $(n=25)$ and two MTC $(n=2)$ isolates (Figure 01) and three MAC $(n=3)$ isolates. Reaction II also confirmed the presence of genus Mycobacterium in addition to the presence of MCAG (Figure 02) in two samples $(n=2)$ (Table 02).

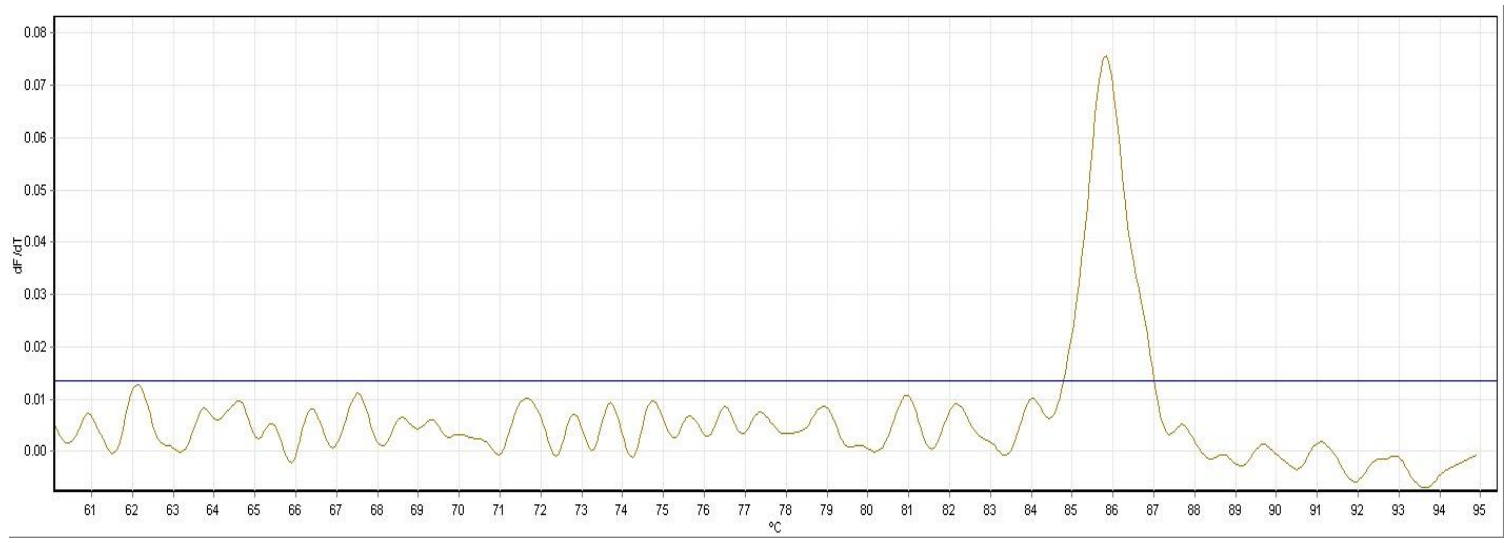

Figure 01: Melting curve generated following the SYBR Green mediated real-time PCR reaction I indication the presence of $M T C(T m=85.80 \mathrm{C})$

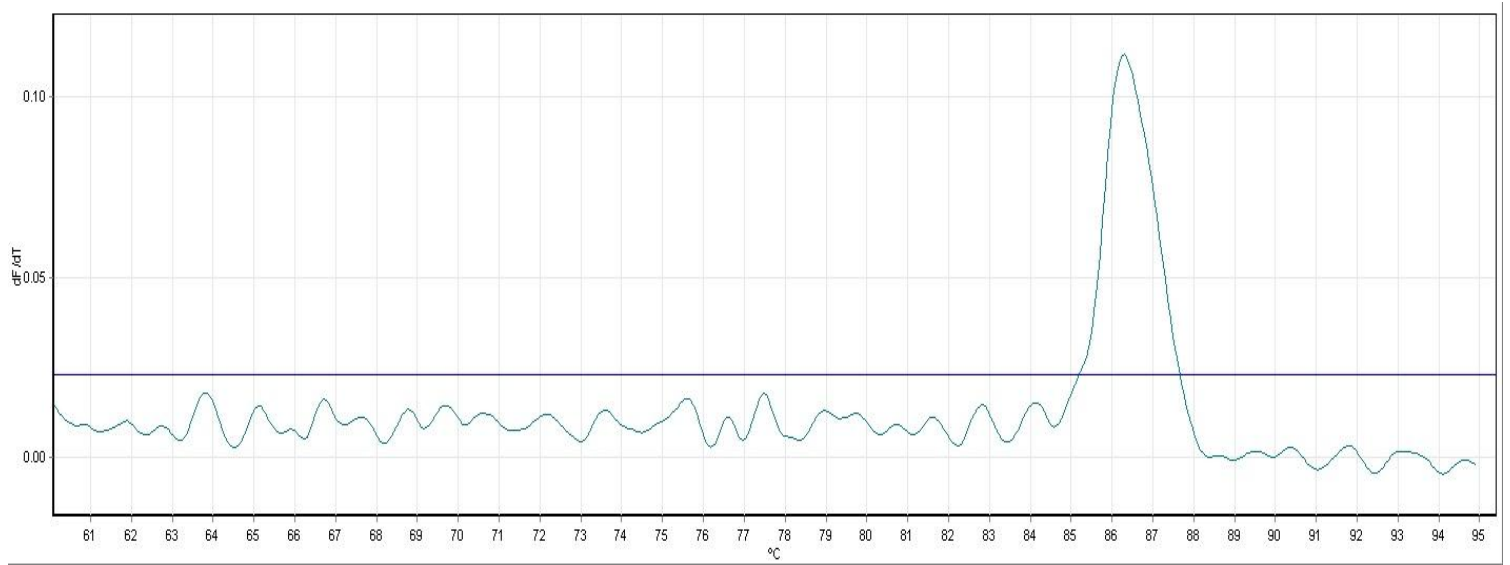

Figure 02: Melting curve generated following the SYBR Green mediated real-time PCR reaction II indication the presence of $M C A G(T m=86.30 C)$ 
Table 02: Number of Mycobacterium isolates identified during the study

\begin{tabular}{lll}
\hline Organism & Reaction & $\begin{array}{l}\text { Number of } \\
\text { Isolates } \\
\text { identified }\end{array}$ \\
\hline $\begin{array}{l}\text { Mycobacterium } \\
\text { genus }\end{array}$ & I / II & 25 \\
MTC & I & 2 \\
MAC & I & 3 \\
MCAG & II & 2 \\
\hline
\end{tabular}

\section{DISCUSSION}

NTM infections are an increasing public health problem in many countries in the world. Limited laboratory facilities and poverty, which may in some cases, lead to unhygienic life style, are significant challenges for infection control approaches in most of the developing countries. Therefore, understanding the trends and true prevalence of NTM is important in controlling the NTM s well as MTB infections.

The early classification of Mycobacteria was based on growth rate, pigmentation, and clinical significance [9]. Mycobacteria can be broadly classified as rapid growers and slow growers [10] Fast growers commonly have two identical copies of the 16S rRNA gene, whereas slow growers are thought to have only one [11]. The isolates identified throughout this study there were 23 Rapid growers while there were only two $(n=2)$ slow growers.

Throughout this study a considerable proportion of the isolates were identified as NTM, which were identified as slender pink rods upon $\mathrm{ZN}$ staining. There are incidences reported, where $M$. celatum isolates were isolated from bronchial washings [12] which is a potential human pathogen [13]. The isolate which was identified upon 16S rRNA sequencing, produced smooth, convex, non-pigmented colonies on L-J medium. Even though this species is identified as slow growing the $M$. celatum isolate identified during this study was found to be rapidly growing which formed colonies on L-J medium in less than 7 days. Increase in incidence of MAC was reported in early 1980's simultaneously with the beginning of the AIDS widespread [14]. During this study three isolates were identified as MAC which was appeared as smooth, opaque colonies. It is believed that the antimicrobial resistance of the MAC is because of the lack of drug penetration due to the complex cell wall structure [15]. Three isolates were also identified as MCAG following reaction II of real time PCR which were also rapidly growing.

In conclusion, Real time PCR assay is a method of excellent sensitivity and specificity. Moreover, the low contamination risk has made real-time PCR technology appealing to be used in the clinical microbiology for diagnostics.

\section{ACKNOWLEDGEMENT}

The authors gratefully acknowledge the National Research Council, Sri Lanka, grant number 11-059 for providing the equipment, Real-Time PCR machine. The corresponding author for the paper, D. N. Magana-Arachchi can be contacted via email at cellbio@ifs.ac.lk .

\section{REFERENCES}

1. Tortoli, E., Impact of genotypic studies on mycobacterial taxonomy: the new mycobacteria of the 1990s. Clin Microbiol Rev, 2003. 16(2): p. 319-54.

2. Ley, S., et al., Non-tuberculous mycobacteria: baseline data from three sites in Papua New Guinea, 2010-2012. Western Pac Surveill Response J, 2015. 6(4): p. 24-9.

3. Hong, Y.J., et al., Usefulness of three-channel multiplex real-time PCR and melting curve analysis for simultaneous detection and identification of the Mycobacterium tuberculosis complex and nontuberculous mycobacteria. Journal of clinical microbiology, 2011. 49(11): p. 39633966.

4. Maiga, M., et al., Failure to recognize nontuberculous mycobacteria leads to misdiagnosis of chronic pulmonary tuberculosis. PLoS One, 2012. 7(5): p. e36902.

5. Dvorska, L., et al., Strategies for differentiation, identification and typing of medically important species of mycobacteria by molecular methods. VETERINARNI MEDICINA-PRAHA-, 2001. 46(11/12): p. 309-328.

6. Espy, M., et al., Real-time PCR in clinical microbiology: applications for routine laboratory testing. Clinical microbiology reviews, 2006. 19(1): p. 165-256.

7. Somerville, W., et al., Extraction of Mycobacterium tuberculosis DNA: a question of containment. J Clin Microbiol, 2005. 43(6): p. 2996-7.

8. Richardson, E.T., D. Samson, and N. Banaei, Rapid Identification of Mycobacterium tuberculosis and nontuberculous mycobacteria by multiplex, real-time PCR. J Clin Microbiol, 2009. 47(5): p. 1497-502.

9. Runyon, E.H., Anonymous mycobacteria in pulmonary disease. The Medical clinics of North America, 1959. 43(1): p. 273-290.

10. García-Agudo, L. and P. García-Martos, Clinical significance and antimicrobial susceptibility of rapidly 
growing mycobacteria. Science against microbial pathogens: communicating current research and technological advances, 2011. 77.

11. Reischl, U., et al., 16S rRNA sequence diversity in Mycobacterium celatum strains caused by presence of two different copies of $16 \mathrm{~S}$ rRNA gene. Journal of clinical microbiology, 1998. 36(6): p. 1761-1764.
12. Couto, I., et al., Identification of nontuberculous mycobacteria in clinical samples using molecular methods: a 3-year study. Clinical Microbiology and Infection, 2010. 16(8): p. 1161-1164.

13. Roxo, E., Philip Suffys, Adalgiza da Silva Rocha and Diogo dos Santos Netto (Fun.

14. Cespedes, M.S. and J.A. Aberg, Mycobacterium avium Complex (MAC).

15. Inderlied, C., C. Kemper, and L. Bermudez, The Mycobacterium avium complex. Clinical microbiology reviews, 1993. 6(3): p. 266. 